\title{
Kasuistiken
}

Rechtsmedizin 2022 $\cdot 32: 36-40$

https://doi.org/10.1007/s00194-021-00467-8

Angenommen: 25. Januar 2021

Online publiziert: 22. Februar 2021

๑ Der/die Autor(en) 2021

\author{
N. Kern ${ }^{1} \cdot$ S. Potente ${ }^{2} \cdot$ M. A. Verhoff ${ }^{1}$ S. C. Kölzer ${ }^{1}$ \\ 'Institut für Rechtsmedizin, Universitätsklinikum Frankfurt, Goethe-Universität, Frankfurt am Main, \\ Deutschland \\ ${ }^{2}$ Institut für Rechtsmedizin, Universität des Saarlandes, Homburg (Saar), Deutschland
}

\section{Kontaktverbrennungen durch einen Nachtspeicherofen - Unfall oder Misshandlung?}

\section{Hintergrund}

Verletzungen durch thermische Einwirkungen in Form von Hitze spielen v. a. im Kindesalter eine große Rolle. In Europa entfällt etwa die Hälfte aller Hospitalisierungen durch hitzebedingte Verletzungen auf Kinder und Jugendliche unter 16 Jahren $[1,2]$, davon sind zwischen 50 und $80 \%$ im Alter unter 5 Jahren [2]. Brandverletzungen stehen nach Autounfällen und Ertrinken an 3. Stelle der zum Tode führenden Unfälle und Verletzungen bei kleinen Kindern [3]. Die häufigsten hitzebedingten Verletzungen im Kindesalter sind Folge von Verbrühungen, gefolgt von Kontaktverbrennungen [4]. Diese stellen $40 \%$ der hitzebedingten Verletzungen bei Kindern unter einem Jahr und werden hauptsächlich durch Haushaltsgegenstände wie erhitzte Metalloberflächen, Haartrockner oder Herdplatten verursacht $[5,6]$. In manchen Fällen ist es möglich, durch die Konfiguration der Verbrennung Rückschlüsse auf den Gegenstand zu ziehen, der die Verletzung verursacht hat [7]. Eine Unterscheidung zwischen einer akzidentellen und intentionellen Entstehung von Verbrühungen oder Verbrennungen kann u. U. mit Schwierigkeiten verbunden sein $[8$, 9]. Bestimmte Verteilungsmuster der Verletzungen sowie Unstimmigkeiten beim berichteten Unfallhergang können Hinweise auf eine mögliche Misshandlung bieten $[10,11]$. Wie wichtig Details bei der Rekonstruktion eines möglichen Unfallhergangs und zur Abgrenzung einer Kindesmisshandlung oder Ver- nachlässigung der Aufsichtspflicht sind, verdeutlicht der dargestellte Fall.

\section{Falldarstellung \\ Vorgeschichte}

Ein ansonsten gesunder, 7 Monate alter, weiblicher Säugling wurde mit Verbrennungen an beiden Beinen von seinen Eltern in der Notaufnahme eines Krankenhauses vorgestellt. Die Eltern hätten sich im Schlafzimmer aufgehalten, als der Säugling, nur mit einem Body bekleidet, im Kinderzimmer gegen einen Nachtspeicherofen gekrabbelt sei und angefangen habe $\mathrm{zu}$ weinen.

\section{Krankenunterlagen}

Klinisch diagnostiziert wurden Kontaktverbrennungen der Grade $2 \mathrm{a}$ und $2 \mathrm{~b}$ an rechtem Oberschenkel, Unterschenkel und Fuß (• Abb. 1) sowie an linkem Unterschenkel und Fuß. Betroffen waren ca. 4-5\% der Körperoberfläche. Es folgte ein 11-tägiger stationärer Aufenthalt, bei dem ein Wunddébridement bzw. am linken Bein eine Wundversorgung mit einem synthetischen Hautersatz (Suprathel $^{\circledR}$, PolyMedics Innovations, Denkendorf, Deutschland) unter Vollnarkose erforderlich waren. Weiterführende konsiliarische Untersuchungen mit einer Ultraschalldiagnostik des Skelettsystems und des Bauches sowie einer Augenhintergrundspiegelung zur Frage einer Kindesmisshandlung ergaben keine Hinweise auf weitere frische oder ältere Verletzungen. Aufgrund des un- 


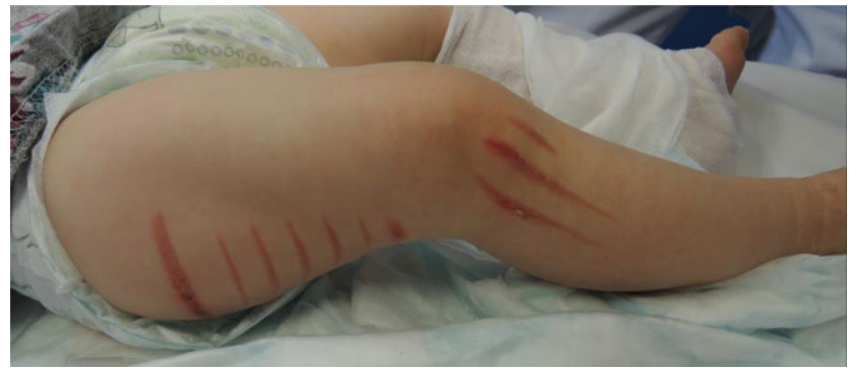

Abb. $1 \Delta$ Streifenförmige Hautverletzungen an der rechten Oberschenkelaußenseite sowie der rechten Knieaußenseite, übergehend auf die rechte Unterschenkelstreckseite (Foto angefertigt in der Klinik, 2 Tage nach dem Ereignis)

klaren Unfallhergangs wurde von den behandelnden Ärzten trotz dessen das Jugendamt eingeschaltet, das eine klinisch-rechtsmedizinische Untersuchung veranlasste.

\section{Klinisch-rechtsmedizinischer Untersuchungsbefund}

Bei der klinisch-rechtsmedizinischen Untersuchung, 10 Tage nach initialer Vorstellung in der Notaufnahme, zeigten sich folgende Hautverletzungen:

- an der Außenseite des rechten Oberschenkels 7 streifenförmige, quer und parallel zueinander verlaufende Hautverletzungen. Die körperstammnächste sowie die körperstammfernste waren jeweils etwa doppelt so hoch wie die 5 dazwischenliegenden Hautbefunde. Die Abstände zwischen den Verletzungen betrugen jeweils etwa $1 \mathrm{~cm}$ (• Abb. 1);

- an der rechten Knievorderaußenseite, übergehend auf die Unterschenkelstreckseite 3, ebenfalls streifenförmige und parallel zueinander gestellte Hautverletzungen mit etwa $1 \mathrm{~cm}$ Abstand zueinander (• Abb. 1);

- eine einzelne weitere, zu den Verletzungen am Oberschenkel sowie der Knievorderaußenseite gleichartige, streifenförmige Hautverletzung an der rechten Unterschenkelrückseite und vereinzelte kurzstreckige Hautverletzungen am Fußrücken mit Einbezug der Zehen;

- an der Außenseite des linken Unterschenkels eine kombinierte, vorwiegend flächige und teils streifenförmige, bereits medizinisch versorgte Hautverletzung (• Abb. 2);
- am äußeren Fußspann links eine flächige und bereits versorgte, kleinere Hautverletzung (• Abb. 3).

\section{Ortsbegehung}

Im Auftrag des zuständigen Jugendamtes erfolgte eine Ortsbegehung der elterlichen Wohnung zur Sichtung der 3 dort befindlichen Nachtspeicheröfen, um die Plausibilität des berichteten Unfallhergangs im Sinne eines Krabbelns gegen den Nachtspeicherofen im Kinderzimmer zu überprüfen.

Hierbei zeigten sich im Wohnzimmer und im elterlichen Schlafzimmer identische Modelle eines Nachtspeicherofens mit einem Luftauslassgitter in Bodennähe mit jeweils 7 , in einem Abstand von $1 \mathrm{~cm}$ zueinander parallel verlaufenden Querstreben (•Abb. 4b). Dabei betrugen die Höhe der obersten und untersten Querstrebe $0,4 \mathrm{~cm}$ und die Höhe der dazwischenliegenden Querstreben jeweils $0,2 \mathrm{~cm}$ (๑Abb. 4). Im Schlafzimmer herrschten zudem beengte Raumverhältnisse, insbesondere der Abstand zwischen Nachtspeicherofen und Ehebett wurde lediglich mit $70 \mathrm{~cm}$ gemessen. Im Kinderzimmer fand sich ein anderes Modell eines Nachtspeicherofens, dessen Luftauslassgitter im Unterschied zu den beiden anderen Öfen 5 parallel verlaufende Querstreben mit ebenfalls jeweils $1 \mathrm{~cm}$ Abstand zueinander aufwies ( $\bullet$ Abb. 4a).

\section{Diskussion}

Die Hautverletzungen an beiden Beinen des Säuglings ließen sich zunächst allein durch die Erklärung der Eltern, das Kind sei gegen den Nachtspeicher-

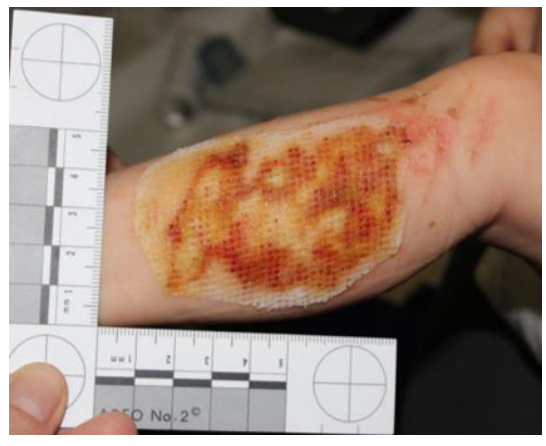

Abb. $2 \Delta$ Kombinierte, vorwiegend flächige, teils auch streifenförmige Hautverletzung an der linken Unterschenkelaußenseite (10 Tage nach dem Ereignis, medizinisch versorgt)

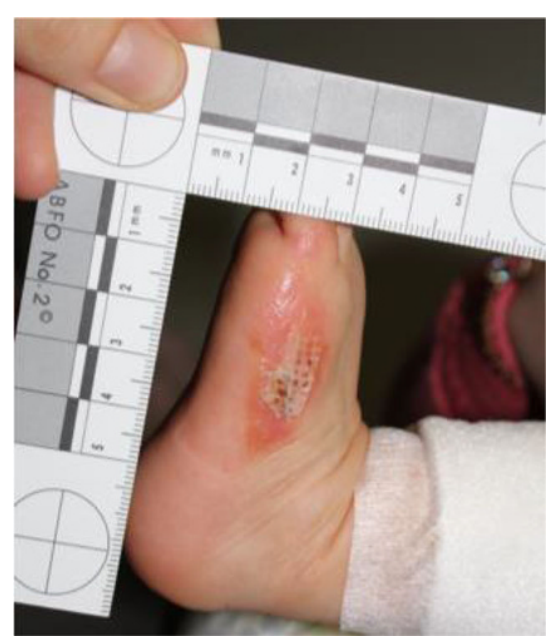

Abb. $3 \Delta$ Kleinflächige Hautverletzung am linken Fußspann (10 Tage nach dem Ereignis, medizinisch versorgt)

ofen im Kinderzimmer gekrabbelt, nicht in Gänze nachvollziehen. Bei unklaren thermischen Verletzungen ist der Anteil an Kindesmisshandlungen bekanntermaßen hoch [12]. Gemäß der Leitlinie Kinderschutz sollte zudem „die Frage nach Vernachlässigung bei jeder thermischen Verletzung als Ursache der Verbrühung/Verbrennung multiprofessionell eingeschätzt und geklärt werden" [12]. Es wurden bis auf die genannten Hautverletzungen keine auffälligen Untersuchungsbefunde wie Knochenbrüche oder andere Verletzungen festgestellt, die gerade in Kombination mit Verbrennungen und Verbrühungen an eine Kindesmisshandlung denken lassen sollten [13]. Bei einer Ortsbegehung ließen sich die Luftauslassgitter der Nachtspeicheröfen in Wohn- und Schlafzimmer mit den Hautverletzungen des Kindes 
in Deckung bringen, jedoch nicht die Luftauslassgitter des Nachtspeicherofens im Kinderzimmer (• Abb. 4). Ergänzend eingeholter technischer Sachverstand ergab, dass im Regelbetrieb bei kalten Außentemperaturen regelmäßig Werte zwischen 80 und $100^{\circ} \mathrm{C}$ am Luftauslassgitter der Nachtspeicheröfen erreicht werden, wobei auch Spitzentemperaturen bis $\mathrm{zu} 150^{\circ} \mathrm{C}$ möglich sind. Laut Literatur [14] reicht bei Erwachsenen bereits ein Kontakt von $1 / 10 \mathrm{~s}$ mit auf $82^{\circ} \mathrm{C}$ erhitzten Flüssigkeiten aus, um Verbrühungen 3. Grades zu verursachen. Die Haut von Säuglingen ist jedoch nur etwa ein Fünftel so dick wie die Haut Erwachsener. Bei ihnen reichen bereits 0,5 s Kontakt mit einer auf $65^{\circ} \mathrm{C}$ erhitzten Flüssigkeit aus, um Verbrennungen 3. Grades hervorzurufen [15]. Tabellen zu Einwirkzeiten bei Kontaktverbrennungen durch verschiedene Feststoffe in Abhängigkeit der Oberflächentemperatur finden sich in der gängigen Literatur nicht. $\mathrm{Zu}$ berücksichtigen ist, dass die meisten Metalle bei gleicher Temperatur und gleichen Druckverhältnissen eine deutlich höhere Wärmeleitfähigkeit als Wasser oder Wasserdampf aufweisen, weshalb bei Kontaktverbrennungen durch Hautkontakt mit einem Metall vereinfacht von einer größeren Wärmeübertragung pro Zeiteinheit und Kontaktfläche im Vergleich zu dem Kontakt mit Wasser oder Wasserdampf anzunehmen ist [16]. Darum kann davon ausgegangen werden, dass bei Säuglingen ein Kontakt der unbekleideten Haut mit dem Luftauslassgitter eines Nachtspeicherofens von deutlich kürzerer Dauer ausreichend ist, um Kontaktverbrennungen wie im dargestellten Fall zu erzeugen. Somit lässt sich ein mögliches Unfallgeschehen wie folgt rekonstruieren: Der Säugling krabbelte mit unbekleideten Beinen (beispielsweise wie angegeben mit einem Body bekleidet) an das Luftauslassgitter des Nachtspeicherofens. Bereits nach einer minimalen Kontaktzeit drehte sich das Kind als Reaktion auf den Schmerzreiz auf den Rücken. Möglicherweise aufgrund der sehr beengten Raumverhältnisse, wie im elterlichen Schlafzimmer vorgefunden, führte dies jedoch dazu, dass das Bein der Gegenseite ebenfalls in Kontakt mit dem heißen

Rechtsmedizin 2022 ·32:36-40 https://doi.org/10.1007/s00194-021-00467-8

(c) Der/die Autor(en) 2021

N. Kern · S. Potente · M. A. Verhoff · S. C. Kölzer

Kontaktverbrennungen durch einen Nachtspeicherofen - Unfall oder Misshandlung?

\section{Zusammenfassung}

Ein 7 Monate alter weiblicher Säugling wurde mit Kontaktverbrennungen 2. Grades an beiden Beinen von seinen Eltern in der Notaufnahme eines Krankenhauses vorgestellt. Die Eltern berichteten, das Kind sei unbeaufsichtigt und nur mit einem Body bekleidet gegen den Nachtspeicherofen im Kinderzimmer gekrabbelt. Bei der 10 Tage später durchgeführten klinisch-rechtsmedizinischen Untersuchung zeigten sich streifige, teils parallel zueinander gestellte und gelenkübergreifende Verbrennungen an der rechten Oberschenkelaußen- und Unterschenkelrückaußenseite, an beiden Fußrücken und den Zehen sowie ein flächenhaftes Verbrennungsareal an der linken Unterschenkelaußenseite mit abgrenzbaren streifigen Anteilen.

Im Rahmen einer Ortsbegehung der elterlichen Wohnung mit Vermessung und Begutachtung der in der Wohnung befindlichen 3 Nachtspeicheröfen konnte zunächst festgestellt werden, dass sich die Verbrennungsmuster an den Beinen des Kindes mit dem Luftauslassgitter der beiden Nachtspeicheröfen im Wohn- und im Elternschlafzimmer (jeweils identisches Modell), hingegen nicht mit dem des Nachtspeicherofens im Kinderzimmer in Deckung bringen ließen. Für die Begutachtung konnte durch ergänzende Informationen eines technischen Sachverständigen zu den entsprechenden Nachtspeicheröfen und durch eine Literaturrecherche ein möglicher Geschehensablauf rekonstruiert werden. Dieser Fall verdeutlicht zum einen, welche Gefahr für Säuglinge und Kleinkinder von Nachtspeicheröfen ausgehen kann, wenn diese nicht regelrecht gesichert und die Kinder unbeaufsichtigt sind. Zum anderen wird die Bedeutung einer detaillierten und - wenn nötig - interdisziplinären Rekonstruktion, inklusive einer Ortsbegehung, zur Abgrenzung eines möglichen Unfallhergangs von einer Kindesmisshandlung unterstrichen.

Schlüsselwörter

Pädiatrische Brandverletzung - Thermische Verletzung · Prävention · Superimposition . Interdisziplinarität

\section{Contact burns from a night storage heater-Accident or abuse?}

\section{Abstract}

A 7-month-old female infant was presented by the parents in the emergency department of a hospital with second-degree contact burns on both legs. The parents reported that the child was unattended and crawled against the night storage stove in the children's room only dressed in a bodysuit. The clinical forensic examination 10 days later revealed striped, partially parallel burns involving multiple joints on the outside of the right thigh and back of the lower leg, on the dorsum of both feet and the toes as well as a large area on the outside of the left lower leg with delimitated striped parts. In the course of an inspection of the parents' apartment with measurement and assessment of the three night storage heaters, it was first determined that the injury patterns on the child's legs matched with the air outlet grid of both night storage heaters in the living room and parents' bedroom (identical models) but not with that of the night storage heater in the children's room. It was possible to reconstruct a potential sequence of events by supplementary information of a technical expert on the corresponding night storage heaters and a literature search. This case illustrates the potential dangers of night storage heaters for unattended infants and toddlers if they are not properly secured and the children are unattended. It also underlines the importance of an interdisciplinary reconstruction, when necessary, including a detailed inspection of the location in order to distinguish a possible accident from child abuse.

\section{Keywords}

Pediatric burn injury - Thermal injury · Prevention · Superimposition · Interdisciplinarity 


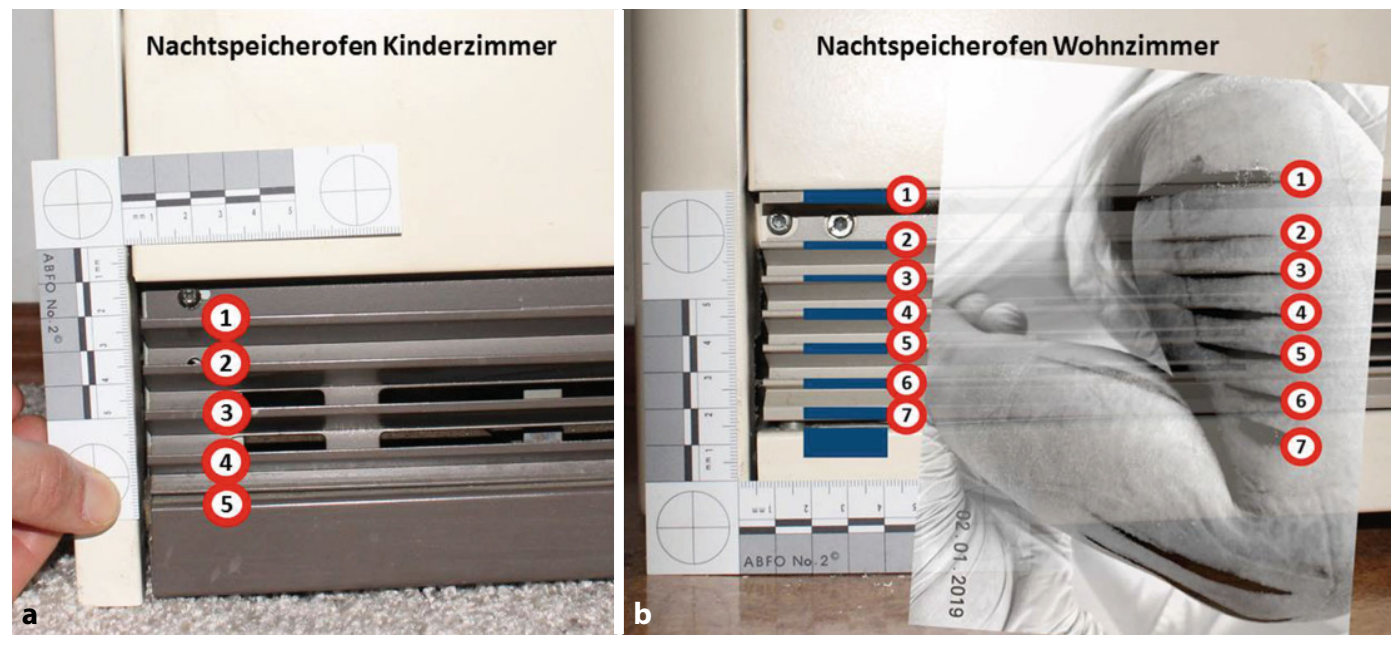

Abb. 4 \ Superimpositionscollage zum Abgleich der Verletzungen an der rechten Oberschenkelaußenseite mit den Luftauslassgittern der Nachtspeicheröfen nach vereinheitlichter Skalierung der verwendeten Abbildungen. Eine Übereinstimmung ist mit den 7 Querstreben des Nachtspeicherofens aus dem Wohnzimmer (baugleich mit dem aus dem Elternschlafzimmer) zu erkennen (b). Der Nachtspeicherofen aus dem Kinderzimmer weist dagegen nur 5 Querstreben auf (a)

Gitter kam, mit hieraus resultierenden weiteren Kontaktverbrennungen.

Die rechtsmedizinischerseits erhobenen Befunde und die resultierenden Schlussfolgerungen hatten zur Folge, dass die Nachtspeicheröfen gegen Kontakt gesichert wurden, um künftig derartigen Unfällen vorzubeugen. Die Erkenntnis, dass das Luftauslassgitter des Nachtspeicherofens im Kinderzimmer nicht zu den im Rahmen der Untersuchung festgestellten Verletzungen passte, offenbarte eine Diskrepanz zwischen den Angaben der Eltern und dem rekonstruierbaren Unfallhergang. Obwohl der Verdacht auf eine Misshandlung entkräftet werden konnte, lag aber zumindest eine Verletzung der Aufsichtspflicht vor. Als Konsequenz wurde eine Unterstützung für die Familie durch das Jugendamt eingerichtet.

\section{Fazit für die Praxis}

Um in dem vorgestellten Fall mit fraglicher Kindesmisshandlung durch thermische Gewalt einen möglichen Geschehensablauf rekonstruieren zu können, waren eine interdisziplinäre Arbeit, einschließlich einer Ortsbegehung, und Literaturrecherche vonnöten. Nur so konnten die Verletzungen mit einem akzidentellen Geschehen in Einklang gebracht werden. Wenngleich sich Diskrepanzen zu den Angaben der Eltern ergaben, konnte der Verdacht auf die Kindesmisshandlung ausgeräumt werden. Weiterhin verdeutlicht der vorliegende Fall die Bedeutung einer Fotodokumentation der Verletzungen zeitnah zum Vorfall durch die behandelnden Ärzte, insbesondere wenn eine klinischrechtsmedizinische Untersuchung erst zeitverzögert erfolgt.

\section{Korrespondenzadresse}

\section{Dr. med. N. Kern}

Institut für Rechtsmedizin, Universitätsklinikum Frankfurt, Goethe-Universität

Kennedyallee 104, 60596 Frankfurt am Main, Deutschland

n.kern@med.uni-frankfurt.de

Funding. Open Access funding enabled and organized by Projekt DEAL.

\section{Einhaltung ethischer Richtlinien}

Interessenkonflikt. N. Kern, S. Potente, M.A. Verhoff und S.C. Kölzer geben an, dass kein Interessenkonflikt besteht.

Für diesen Beitrag wurden von den Autoren keine Studien an Menschen oder Tieren durchgeführt. Für die aufgeführten Studien gelten die jeweils dort angegebenen ethischen Richtlinien. Für Bildmaterial oder anderweitige Angaben innerhalb des Manuskripts, über die Patienten zu identifizieren sind, liegt von innen und/oder ihren gesetzlichen Vertretern eine schriftliche Einwilligung vor.

Open Access. Dieser Artikel wird unter der Creative Commons Namensnennung 4.0 International Lizenz veröffentlicht, welche die Nutzung, Vervielfältigung, Bearbeitung, Verbreitung und Wiedergabe in jeglichem Medium und Format erlaubt, sofern Sie den/die ursprünglichen Autor(en) und die Quelle ordnungsgemäß nennen, einen Link zur Creative Commons Lizenz beifügen und angeben, ob Änderungen vorgenommen wurden.

Die in diesem Artikel enthaltenen Bilder und sonstiges Drittmaterial unterliegen ebenfalls der genannten Creative Commons Lizenz, sofern sich aus der Abbildungslegende nichts anderes ergibt. Sofern das betreffende Material nicht unter der genannten Creative Commons Lizenz steht und die betreffende Handlung nicht nach gesetzlichen Vorschriften erlaubt ist, ist für die oben aufgeführten Weiterverwendungen des Materials die Einwilligung des jeweiligen Rechteinhabers einzuholen.

Weitere Details zur Lizenz entnehmen Sie bitte der Lizenzinformation auf http://creativecommons.org/ licenses/by/4.0/deed.de.

\section{Literatur}

1. Kemp AM, Jones S, Lawson Z et al (2014) Patterns of burns and scalds in children. Arch Dis Child 99(4):316-321

2. Brusselaers N, Monstrey S, Vogelaers D (2010) Severe burn injury in Europe: a systematic review of the incidence, etiology, morbidity, and mortality. Crit Care 14(5):R188

3. Toon MH, Maybauer DM, Arceneaux LL et al (2011)Children with burn injuries - assessment of trauma, neglect, violence and abuse. J Inj Violence Res 3(2):98-110

4. Laitakari E, Koljonen V, Pyörälä S (2014) Outpatient treated burns in infants younger than 1 year in Helsinki during 2005-2009. Burns 40(3):489-494

5. Yen KL, BankDE, O'Neill AM et al (2001) Household oven doors: a burn hazard in children. Arch Pediatr Adolesc Med 155(1):84-86

6. Johnson EL, Maguire S, Hollén LI et al (2017) Agents, mechanisms and clinical features of non- 


\section{Kasuistiken}

scald burns in children: a prospective UK study. Burns 43(6):1218-1226

7. Hobbs CJ (1986) When are burns not accidental? Arch Dis Child 61(4):357-361

8. Pawlik MC, Kemp A, Maguire Set al (2016) Children with burns referred for child abuse evaluation: burn characteristics and co-existent injuries. Child Abuse Negl 55:52-61

9. Rosado N, Charleston E, Gregg M (2019) Characteristics of accidental versus abusive pediatric burn injuries in an urban burn center over a 14 year period. J Burn Care Res. 40(4):437-443. https://doi. org/10.1093/jbcr/irz032

10. Mullen S, Begley R, Roberts Z et al (2018) Fifteenminute consultation: childhood burns: inflicted, neglect or accidental. Arch Dis Child Educ Pract Ed 104(2):74-78

11. Peck MD, Priolo-Kapel D (2002) Child abuse by burning: a review of the literature and an algorithm for medical investigations. J Trauma 53(5):1013-1022

12. 027-069l_S3_Kindesmisshandlung-missbrauchvernachlaessigung-Kinderschutzleitlinie_201902_1_01.pdf; Zugegriffen: 9.Jan. 2021

13. Wibbenmeyer L, Liao J, Heard J et al (2014) Factors related to child maltreatment in children presenting with burn injuries. J Burn Care Res 35(5):374-381

14. Abraham JP, Plourde B, Vallez L et al (2015) Estimating the time and temperature relationship for causation of deep-partial thickness skin burns. Burns 41(8):1741-1747

15. Herrmann B, Dettmeyer R, Banaschak Set al (2016) Kindesmisshandlung - Medizinische Diagnostik, Intervention und rechtliche Grundlagen, 3. Aufl. Springer, Berlin Heidelberg, 579

16. Böckh P, Wetzel T (2018) Wärmeübertragung: Grundlagen und Praxis. Springer, Berlin Heidelberg, S 10

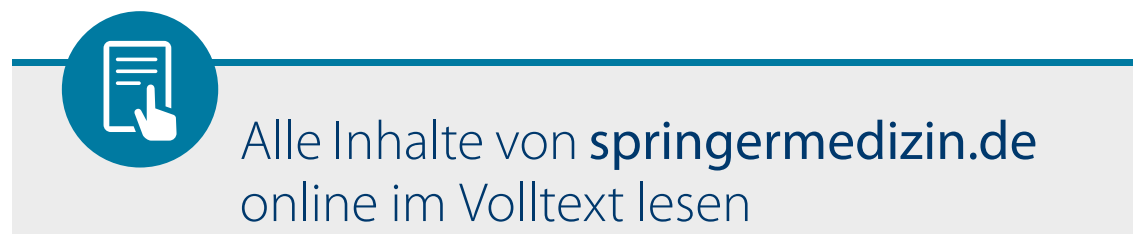

Mit e.Med Interdisziplinär - dem Kombi-Abo von SpringerMedizin.de können Sie jederzeit auf alle Inhalte zugreifen, die Sie für Ihren Praxis- oder Klinikalltag benötigen. Sie sind immer aktuell informiert - über die neuesten Publikationen, wichtige Studien oder innovative Therapieverfahren.

Die Vorteile von e.Med Interdisziplinär:

- Gestalten Sie Ihre Fortbildung nach Ihren Bedürfnissen: e.Med Interdisziplinär bietet Ihnen Online-Zugang zu 600 CME-Fortbildungskursen aller Fachrichtungen

- Sie entscheiden, was Sie lesen möchten:

Alle Fachzeitschriften stehen digital im Volltext zur Verfügung.

- Finden Sie die gewünschten Informationen auch in englischen Publikationen: Sie können in mehr als 500 englischsprachigen Fachzeitschriften online recherchieren und auf die Suchergebnisse uneingeschränkt im Volltext zugreifen.

- Auf Wunsch erhalten sie darüber hinaus eine gedruckte Fachzeitschrift nach Wahl.

Mit den fachspezifischen e.Med-Abos können Sie sich effizient innerhalb Ihres Fachgebietes auf dem Laufenden halten. Das Angebot reicht von AINS bis Radiologie - Sie haben die Wahl!

Testen Sie e.Med kostenlos und unverbindlich!

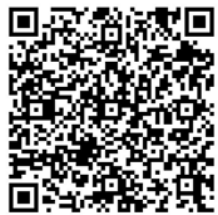
Jetzt informieren unter www.springermedizin.de $\Rightarrow$ "Abos"

oder telefonisch unter 0800-77 80777

(Montag bis Freitag, 10 bis $17 \mathrm{Uhr}$ ) 